\title{
Myocarditis Caused by Human Parechovirus in Adult
}

\section{Khai Lin Kong, Jillian S.Y. Lau, Su Mei Goh, Heather L. Wilson, Mike Catton, Tony M. Korman}

The infectious etiology of myocarditis often remains unidentified. We report a case of myocarditis associated with human parechovirus (HPeV) infection in an adult. HPeV is an emerging pathogen that can cause serious illness, including myocarditis, in adults. Testing for HPeV should be considered in differential diagnosis of myocarditis.

Tnfections with human parechovirus $(\mathrm{HPeV})$ are rarely reported in adults. We report a case of myocarditis associated with $\mathrm{HPeV}$ infection in an adult.

\section{The Study}

During the summer of 2015, a 26-year-old man in Victoria, Australia, was admitted to Casey Hospital (Berwick, VIC, Australia) because of 4 days of fever, rigors, headache, dry cough, sore throat, myalgia, and a history of erythematous macular rash on arms bilaterally that had resolved by the time of admission. The patient smoked cigarettes and reported use of methamphetamines, but no other medical history was reported. He lived in a rural area but had no close contact with animals. He lived with 3 young children, including an 8-week-old infant who had recently had otitis externa.

At admission, he was febrile (temperature $38.2^{\circ} \mathrm{C}$ ) and had sinus tachycardia $(\leq 130$ beats/min). Results of a physical examination were otherwise unremarkable. Peripheral blood lymphocyte count was $0.70 \times 10^{9}$ cells $/ \mathrm{L}$ (reference range $1-4 \times 10^{9}$ cells $/ \mathrm{L}$ ), C-reactive protein level $111 \mathrm{mg} / \mathrm{L}$ (reference value $<5 \mathrm{mg} / \mathrm{L}$ ), erythrocyte sedimentation rate $94 \mathrm{~mm} / \mathrm{h}$ (reference value $<10 \mathrm{~mm} / \mathrm{h}$ ), serum bilirubin level $49 \mu \mathrm{mol} / \mathrm{L}$ (reference value $<20 \mu \mathrm{mol} / \mathrm{L}$ ), and albumin level $24 \mathrm{~g} / \mathrm{L}$ (reference range 35-45 g/L).

Microscopic analysis of cerebrospinal fluid (CSF) showed $2 \times 10^{6}$ polymorphonuclear cells/L, $2 \times 10^{6}$ lymphocytes/L, a total protein level of $0.5 \mathrm{~g} / \mathrm{L}$ (reference range $0.1-0.3 \mathrm{~g} / \mathrm{L}$ ), and glucose and lactate levels within reference ranges. Blood and CSF cultures showed

Author affiliations: Monash University, Clayton, Victoria, Australia (K.L. Kong, J.S.Y. Lau, S.M. Goh, T.M. Korman); Monash Health, Clayton (K.L. Kong, J. Lau, S.M. Goh, T.M. Korman); Peter Doherty Institute for Infection and Immunity, Melbourne, Victoria, Australia (H.L. Wilson, M. Catton)

DOI: https://doi.org/10.3201/eid2309.161256 no bacterial growth. Because of a low leukocyte count, molecular studies for viruses (including enterovirus) were not performed for the CSF sample.

Fever and tachycardia persisted for 5 days and chest discomfort and dyspnea developed. A transthoracic echocardiogram showed a mildly dilated left ventricle with an ejection fraction of $15 \%$. There were no valvular vegetations. Peak creatine kinase level was $713 \mathrm{U} / \mathrm{L}$ (reference value $<230 \mathrm{U} / \mathrm{L}$ ), and troponin level was $15.28 \mu \mathrm{g} / \mathrm{L}$ (reference value $<0.080 \mu \mathrm{g} / \mathrm{L}$ ).

The patient was given intravenous benzylpenicillin and oral doxycycline as empirical therapy for possible bacterial infection; Q fever and leptospirosis were considered possible diagnoses. Fever and chest discomfort improved, and he was discharged 7 days after admission. Two weeks later, the patient was well and had minimal dyspnea.

Throat swab specimens were obtained on day 6 of illness, and rectal swab specimens were obtained on day 8 of illness. Specimens were tested for enterovirus and $\mathrm{HPeV}$ RNA by reverse transcription PCR (RT-PCR) and primers specific for the highly conserved $5^{\prime}$ untranslated region (1) (details for $\mathrm{HPeV}$ primers and probes are available on request). HPeV was detected in the throat swab specimen, but not the rectal swab specimen.

We attempted molecular typing of $\mathrm{HPeV}$ by using the method of Papadakis et al. (1) and primers AN353, AN355, AN357, AN358, and AN369 described by Nix et al. (2). However, typing was not successful because of low copy numbers, probably caused by specimens being collected late in the illness.

Multiple investigations showed no other infectious causes of myocarditis. Serologic results were negative for previous or recent infections with hepatitis $\mathrm{A}, \mathrm{B}$, and $\mathrm{C}$ viruses and HIV, as well as Leptospira spp., Coxiella burnetii, rickettsia, Treponema pallidum, and Toxoplasma spp. Serologic analysis showed evidence of previous infections with cytomegalovirus and Epstein-Barr virus. However, a convalescent-phase serum sample was not available for additional serologic testing.

A multiplex PCR (Respiratory Pathogens B; AusDiagnostics, Beaconsfield, NSW, Australia) was performed for a nasopharyngeal swab specimen. Results were negative for influenza A virus; A(H1N1)pdm09 virus; influenza B virus; respiratory syncytial virus; rhinoviruses/enterovirus; human parainfluenza virus 1, 2, and 3; adenovirus (groups B, C, E, some A, D); human metapneumovirus; Bordetella 
pertussis and B. parapertussis; Legionella pneumophila and L. longbeachae; Mycoplasma pneumoniae; and Chlamydia/Chlamydophila spp. (including C. psittaci, C. pneumoniae, and C. trachomatis).

\section{Conclusions}

$\mathrm{HPeVs}$ were previously classified as a subgenus of echoviruses (3). Echovirus subtypes 22 and 23 were renamed $\mathrm{HPeV}$ type 1 and 2; sixteen different types of $\mathrm{HPeV}$ thus far have been identified. Serosurveillance studies showed that by 2 years of age, $\leq 90 \%$ of children are infected with $\geq 1$ type of $\mathrm{HPeV}$ (3). Infections with human parechoviruses show various clinical manifestations, notably sepsis-like disease and encephalitis in infants. A recent large outbreak of $\mathrm{HPeV}$ type 3 infections in infants was reported in Australia (4).

$\mathrm{HPeV}$ infections in adults are rarely reported. Mizuta et al. reported 22 adults with myalgia, muscular weakness, sore throat, orchiodynia, and increased levels of creatine phosphokinase; 14 had HPeV type 3 infections confirmed by virus isolation, positive RT-PCR results for throat swab or stool specimens, or serologic analysis (5). $\mathrm{HPeV}$ was also reported to be associated with flaccid paralysis and diarrheal illness in adults $(6,7)$.

The rarity of $\mathrm{HPeV}$ infection in adults could be related to immunity conferred by previous exposure during childhood to $\mathrm{HPeV}$. Few seroprevalence data are available for $\mathrm{HPeV}$ infections in adults. However, as part of an investigation of infant deaths associated with $\mathrm{HPeV}$ type 3 in Wisconsin, USA, limited serologic testing of 59 adults demonstrated that infections were not common, suggesting that either $\mathrm{HPeV} 3$ was a new pathogen being introduced to this community, or that there was waning immunity, which made antibody titers difficult to detect in adults (8).

The lack of documented reports of $\mathrm{HPeV}$ infection in adults could also be caused by lack of widespread testing for adults. HPeV RNA is not detected by routine enterovirus PCRs and requires additional $\mathrm{HPeV}$ testing. The Victorian
Infectious Diseases Reference Laboratory (Melbourne, VIC, Australia) routinely tests specimens for enterovirus and $\mathrm{HPeV}$ when a request is made, regardless of the age of the patients. During January 2015-May 2016, this laboratory tested 3,525 specimens for $\mathrm{HPeV}$, of which 1,425 (40\%) were obtained from adults. HPeV was detected by RT-PCR in $5(0.35 \%)$ of 1,425 specimens: 2 in throat swab specimens, 2 in blood, and 1 in CSF. In comparison, 286 $(13.6 \%)$ of 2,100 specimens from persons $<18$ years of age were positive for $\mathrm{HPeV}$; most $(271,94.8 \%$ ) were from children $<1$ year of age. This finding suggests that, although increased testing for $\mathrm{HPeV}$ could increase the detection rate of $\mathrm{HPeV}$ infection in adults, it is an uncommon infection in the adult population. This finding is consistent with results of a study from a reference laboratory in Scotland that tested 3,739 CSF samples from persons of all ages and found that although enteroviruses were common in adults, $\mathrm{HPeV}$ infections were found exclusively in young infants (9).

Enteroviruses are recognized as a major cause of acute myocarditis and are associated with $\leq 14 \%$ of cases (10). Myocarditis associated with $\mathrm{HPeV}$ infections is rarely reported (Table). This disease has been reported in 3 children $<2$ years of age and 1 adolescent. Two of the patients were immunosuppressed, 1 of whom died. A study of 109 patients infected with echovirus 22 (now HPeV subtype 1) in Sweden included a case of myocarditis in a child; virus was isolated from a stool sample and a major increase in antibody titer was observed (11).

There is no proven effective therapy for $\mathrm{HPeV}$ infection. Intravenous immunoglobulin (IVIG) was used for 2 patients (Table). IVIG has been used for treatment of enterovirus infections, particularly in immunocompromised patients (15), but the efficacy of IVIG might be limited for treatment of $\mathrm{HPeV}$ infection because of low seroprevalence in adults $(8)$.

In summary, we report a case of myocarditis associated with $\mathrm{HPeV}$ infection in an adult. A large proportion of cases of myocarditis has no identified infectious cause.

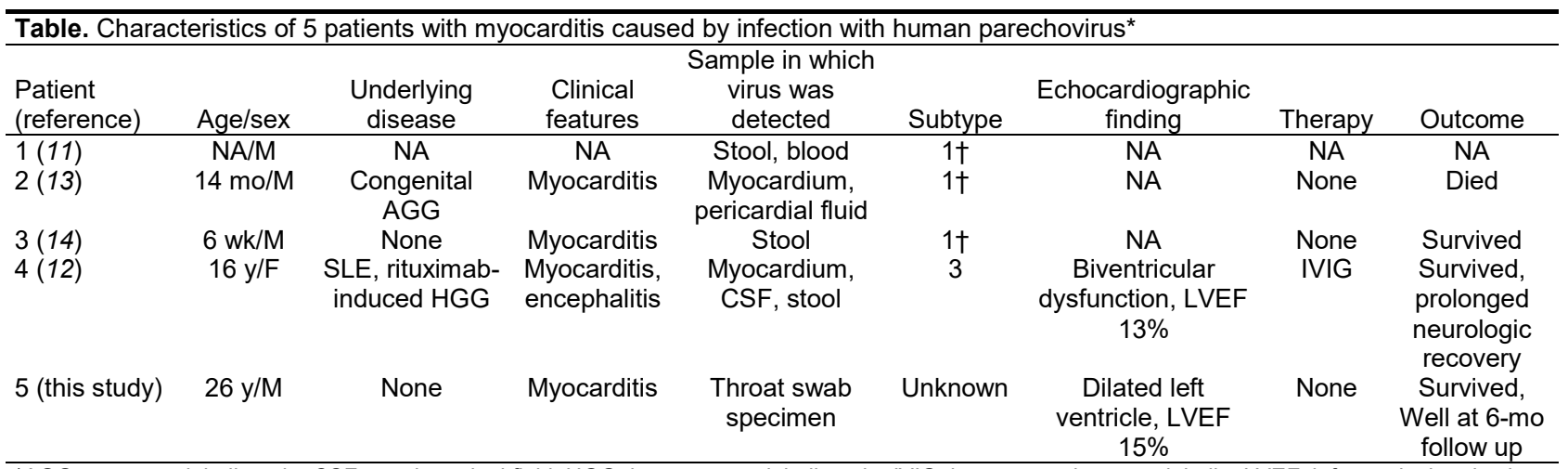

*AGG, agammaglobulinemia; CSF, cerebrospinal fluid; HGG, hypogammaglobulinemia; IVIG, intravenous immunoglobulin; LVEF, left ventricular ejection fraction; NA, not available; SLE, systemic lupus erythematosus.

†Previously known as echovirus subtype 22. 
Thus, testing of throat swab, stool, and blood specimens for $\mathrm{HPeV}$ should be considered for adults with myocarditis. $\mathrm{HPeV}$ is an emerging pathogen that can cause major illness, including myocarditis, in adults.

Dr. Kong is an infectious diseases fellow at Monash Health, Melbourne, Victoria, Australia. His primary research interest is emerging virus infections.

\section{References}

1. Papadakis G, Tsortos A, Kordas A, Tiniakou I, Morou E, Vontas J, et al. Acoustic detection of DNA conformation in genetic assays combined with PCR. Sci Rep. 2013;3:2033. http://dx.doi.org/ 10.1038/srep02033

2. Nix WA, Maher K, Pallansch MA, Oberste MS. Parechovirus typing in clinical specimens by nested or semi-nested PCR coupled with sequencing. J Clin Virol. 2010;48:202-7. http://dx.doi.org/10.1016/j.jcv.2010.04.007

3. de Crom SCM, Rossen JWA, van Furth AM, Obihara CC. Enterovirus and parechovirus infection in children: a brief overview. Eur J Pediatr. 2016;175:1023-9. http://dx.doi.org/ 10.1007/s00431-016-2725-7

4. Khatami A, McMullan BJ, Webber M, Stewart P, Francis S, Timmers KJ, et al. Sepsis-like disease in infants due to human parechovirus type 3 during an outbreak in Australia. Clin Infect Dis. 2015;60:228-36. http://dx.doi.org/10.1093/cid/ciu784

5. Mizuta K, Kuroda M, Kurimura M, Yahata Y, Sekizuka T, Aoki Y, et al. Epidemic myalgia in adults associated with human parechovirus type 3 infection, Yamagata, Japan, 2008. Emerg Infect Dis. 2012;18:1787-93. http://dx.doi.org/10.3201/eid1811.111570

6. Saikruang W, Khamrin P, Suantai B, Okitsu S, Hayakawa S, Ushijima $\mathrm{H}$, et al. Detection of diarrheal viruses circulating in adult patients in Thailand. Arch Virol. 2014;159:3371-5. http://dx.doi.org/10.1007/s00705-014-2191-3

7. Figueroa JP, Ashley D, King D, Hull B. An outbreak of acute flaccid paralysis in Jamaica associated with echovirus type
22. J Med Virol. 1989;29:315-9. http://dx.doi.org/10.1002/ jmv. 1890290418

8. Sedmak G, Nix WA, Jentzen J, Haupt TE, Davis JP, Bhattacharyya S, et al. Infant deaths associated with human parechovirus infection in Wisconsin. Clin Infect Dis. 2010; 50:357-61. http://dx.doi.org/10.1086/649863

9. Harvala H, McLeish N, Kondracka J, McIntyre CL, McWilliam Leitch EC, Templeton K, et al. Comparison of human parechovirus and enterovirus detection frequencies in cerebrospinal fluid samples collected over a 5-year period in Edinburgh: HPeV type 3 identified as the most common picornavirus type. J Med Virol. 2011;83:889-96. http://dx.doi.org/ 10.1002/jmv.22023

10. Knowlton KU, Narexkina A, Savoia MC, Oxman MN. Myocarditis and pericarditis. In: Bennett JE, Dolin R, Blaser MJ, editors. Mandell, Douglas, and Bennett's principles and practice of infectious diseases. 8th ed. Amsterdam: Elsevier; 2015. p. 1066-79.

11. Ehrnst A, Eriksson M. Epidemiological features of type 22 echovirus infection. Scand J Infect Dis. 1993;25:275-81. http://dx.doi.org/10.3109/00365549309008499

12. Mardekian SK, Fortuna D, Nix A, Bhatti T, Wiley CA, Flanders A, et al. Severe human parechovirus type 3 myocarditis and encephalitis in an adolescent with hypogammaglobulinemia. Int J Infect Dis. 2015;36:6-8. http://dx.doi.org/10.1016/j.ijid.2015.05.008

13. Maller HM, Powars DF, Horowitz RE, Portnoy B. Fatal myocarditis associated with ECHO virus, type 22, infection in a child with apparent immunological deficiency. J Pediatr. 1967;71:204-10. http://dx.doi.org/10.1016/S0022-3476(67)80073-8

14. Russell SJ, Bell EJ. Echoviruses and carditis. Lancet. 1970;1:784 5. http://dx.doi.org/10.1016/S0140-6736(70)91021-4

15. Dendle C, Gilbertson M, Korman TM, Golder V, Morand E, Opat S. Disseminated enteroviral infection associated with obinutuzumab. Emerg Infect Dis. 2015;21:1661-3. http://dx.doi.org/10.3201/eid2109.150104

Address for correspondence: Khai Lin Kong, Department of Infectious Diseases, Monash Health, 246 Clayton Rd, Clayton VIC 3168, Australia: email: kong.khailin@gmail.com

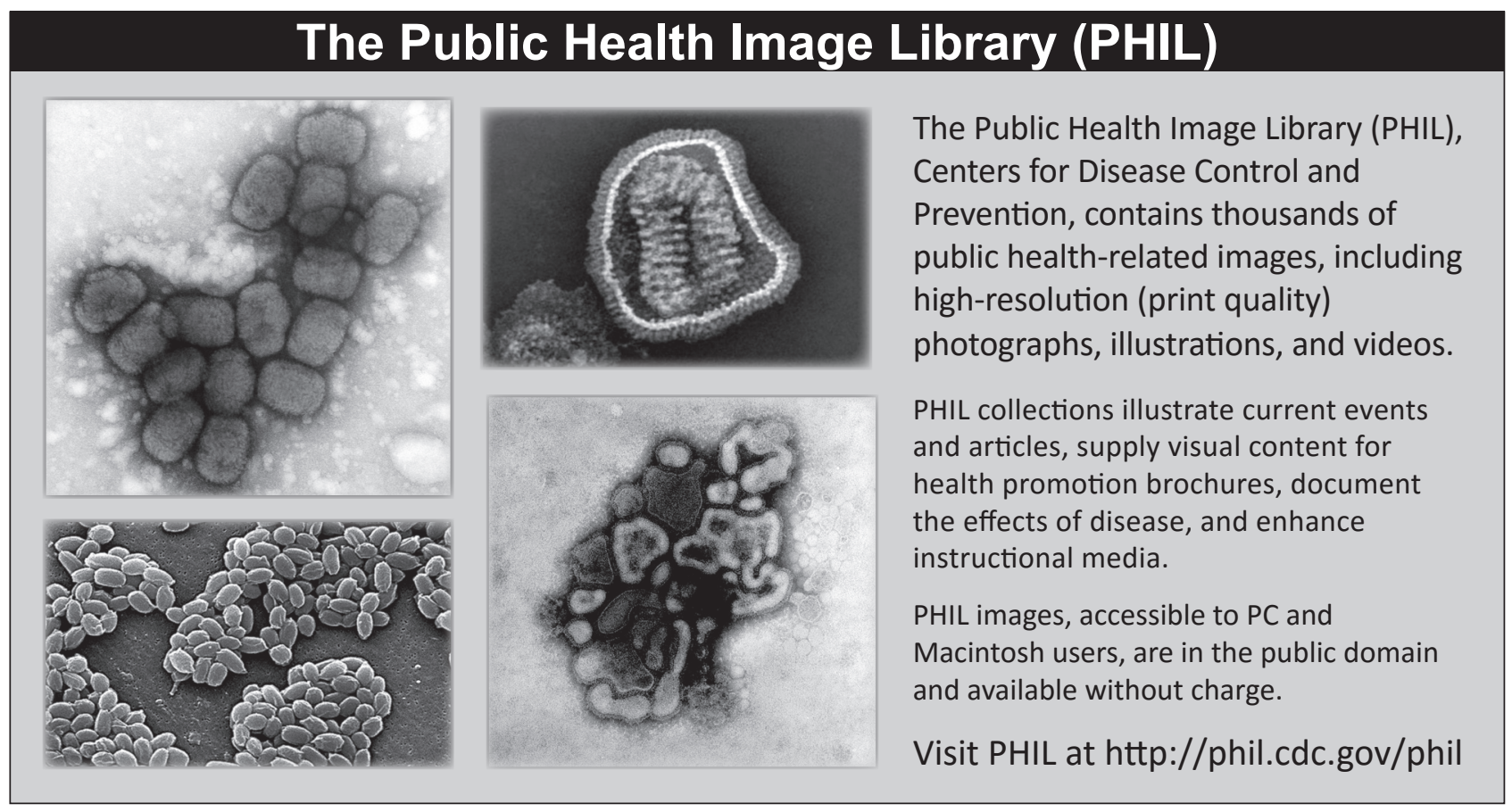

